\title{
TEMPERATURES IN A THIN METAL PLATE TRAVERSED BY AN ARC
}

\author{
BY \\ R. L. BISH \\ Materials Research Laboratories, Ascot Vale, Victoria, Australia
}

\begin{abstract}
The temperature distribution within a thin metal plate of infinite extent traversed by an electric arc is obtained for the case when the plate remains electronegative. The potential application of this result to the experimental determination of the size of the cathode dark space is discussed.
\end{abstract}

1. Introduction. A problem that arises in attempts to characterise the high pressure metallic arc, used in welding and for illumination, is that of determining the size of the cathode dark space in the arc at the electronegative gas/metal junction. No method has yet been devised that can be used to accurately determine the extent of this region [1]; despite the fact that it exerts considerable influence over the temperatures prevailing at the arc spot [2].

A naturally occurring idea, however, is that, if the temperatures in a plate traversed by an arc can be derived analytically then, by comparing theoretical and measured temperatures, the extent of the dark space, figuring in the analytic solution, may be calculated.

At the cathode spot only arc radiation seems to be responsible for the heating of the electrode; and in this paper the relationship connecting the cathode dark space size and the cathode spot temperature is derived. In order to do this it becomes necessary to modify the Wilson-Rosenthal $[3,4]$ moving heat source theory; so as to make allowance for the distributed character of arc radiation.

2. Moving heat source theory. It proves convenient to treat the arc as stationary, and to consider a closed surface, $S$, within the cathode but fixed in space; so that the plate material passes through $S$. Then, due to mass movement, there will be a heat flux across $S$, the density of which equals $\rho c v \Phi \mathbf{n}$, where $\rho$ is the mass density, $c$ the specific heat, $v$ the speed, and $\Phi$ the temperature in the plate, while $\mathbf{n}$ is a unit vector directed parallel to the plate movement. In addition there will be a heat flux due to conduction along the negative temperature gradient in the space fixed relative to the arc spot. Denoting the density of this conducted flux by $\mathbf{J}$, and the volume enclosed by $S$ by $V$, it follows from the conservation of heat energy that, if $Q$ is 
the rate of heat production per unit volume,

$$
\int_{S}(\mathbf{J}+\rho c v \Phi \mathbf{n}) \cdot d \mathbf{S}=\int_{V} Q d V .
$$

Transforming the integral on the left, by means of Gauss' divergence theorem, and using the rule for the divergence of a product of a scalar and a vector [5], we obtain

$$
\operatorname{div} \mathbf{J}+\rho c v \mathbf{n} \cdot \operatorname{grad} \Phi=Q .
$$

But, by Fourier's law,

$$
\mathbf{J}=-\kappa \operatorname{grad} \Phi,
$$

where $\kappa$ is the thermal conductivity of the plate. Moreover, if $\sigma$ is the distance that a point lies from the arc spot centre, measured in the direction $-\mathbf{n}$ (Fig. 1) then,

$$
\mathbf{n} \cdot \operatorname{grad} \Phi=-\frac{\partial \Phi}{\partial \sigma}
$$

and, on substituting for $\mathbf{J}$ from Fourier's law and using this result in Eq. (1), we get

$$
\nabla^{2} \Phi+2 \lambda v \frac{\partial \Phi}{\partial \sigma}=-Q / \kappa
$$

where

$$
2 \lambda \kappa=\rho c .
$$

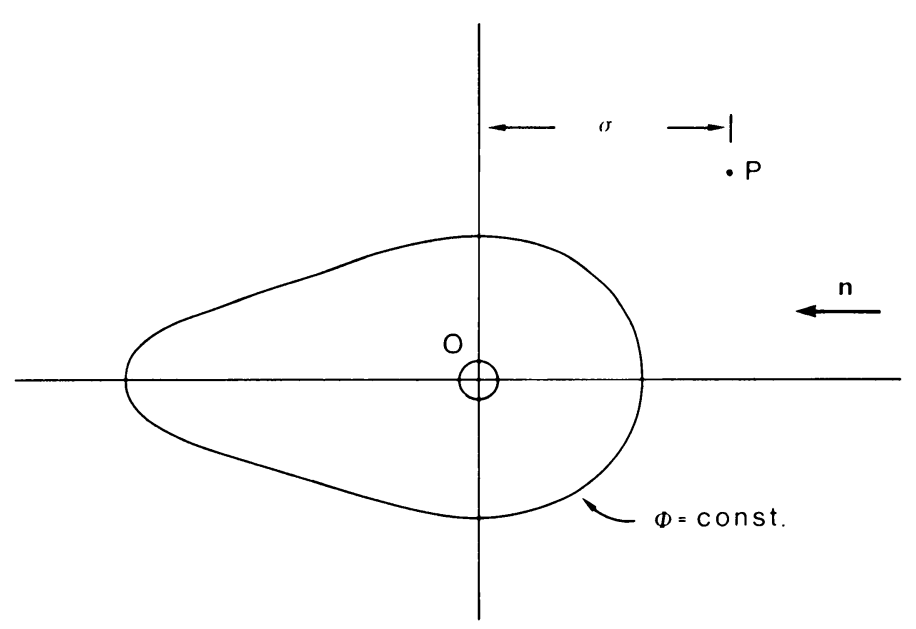

Fig. 1. The isotherms in the space occupied by the plate, as viewed from the (stationary) arc exhibit the shape shown schematically; a point in this space lies at distance $\sigma$ from the arc, measured opposite to the sense of apparent plate movement.

In the case of a thin plate the heat flow problem becomes two dimensional and for $Q$ we write $W / h$, where $W$ is the normal heat flux density on the plate surface due to arc radiation, and where $h$ is the plate thickness. In this case Eq. (2) becomes

$$
\nabla^{2} \Phi+2 \lambda v \frac{\partial \Phi}{\partial \sigma}=-W / \kappa h
$$


In order to reduce this equation to a recognisable form we must make the substitution [4]

$$
\Phi=e^{-\lambda v \sigma} \phi
$$

where $\phi$ is a function of the coordinates in the plane of the plate, with origin at the arc spot centre. The equation thus obtained is

$$
\nabla^{2} \Phi-\lambda^{2} v^{2} \Phi=-\frac{W}{\kappa h} e^{\lambda v \sigma}
$$

which is related to the Helmholtz equation [6].

3. Integral solution. To solve Eq. (5) we apply Green's theorem in the form

$$
\int_{S}\left(\phi^{\prime} \nabla^{2} \phi-\phi \nabla^{2} \phi^{\prime}\right) d S=\int_{C}\left(\phi^{\prime} \frac{\partial \phi}{\partial n}-\phi \frac{\partial \phi^{\prime}}{\partial n}\right) d s,
$$

where $S$ is now any area in the plane of the plate bounded by a closed curve $C$, along which $s$ measures distance, and normal to which $\partial / \partial n$ denotes differentiation in the direction of the outward drawn normal; while $\phi$ and $\phi^{\prime}$ are any two functions which, along with their derivatives, remain continuous in $S$. We assume that $\phi$ satisfies Eq. (5) while

$$
\nabla^{2} \phi^{\prime}-\lambda^{2} v^{2} \phi^{\prime}=0
$$

and we seek a solution for a plate with its boundaries at infinity. From Eqs. (5), (6), and (7) we get

$$
\int_{S}-\frac{W}{\kappa h} \phi^{\prime} e^{\lambda n \sigma} d S=\int_{C}\left(\phi^{\prime} \frac{\partial \phi}{\partial n}-\phi \frac{\partial \phi^{\prime}}{\partial n}\right) d s .
$$

Further, a solution to Eq. (7) is

$$
\phi^{\prime}=K_{0}(\lambda \rho v),
$$

where $K_{0}$ denotes the modified Bessel function of the second kind and zero order, and $\rho$ is radial distance in the plane of the plate from the pole $P$, at which we seek the value of $\phi$ (Fig. 2). Since $\phi^{\prime}$ now becomes infinite as $\rho \rightarrow 0$, we must deform $C$ to consist of a small circle $C_{1}$, described counterclockwise about $P$, and a circle $C_{2}$ at infinity. With these changes Eq. (8) becomes, on letting the radius of $C_{1}$ tend to zero,

$$
\frac{1}{\kappa h} \int_{S} W K_{0}(\lambda \rho v) e^{\lambda \nu \sigma} d S=\lim _{\rho \rightarrow 0} 2 \pi \rho\left(-K_{0}(\lambda \rho v) \frac{\partial \phi}{\partial \rho}+\phi \frac{\partial}{\partial \rho} K_{0}(\lambda \rho v)\right) .
$$

But we know that [7]

$$
\begin{gathered}
\lim _{t \rightarrow 0} K_{0}(t)=-I_{0}(t) \ln (t / 2), \\
\lim _{t \rightarrow 0} t K_{0}^{\prime}(t)=-1,
\end{gathered}
$$

where $I_{0}$ is the modified Bessel function of the first kind of zero order, and so

$$
2 \pi \phi=\frac{1}{\kappa h} \int_{S} W K_{0}(\lambda \rho v) e^{\lambda n \sigma} d S
$$




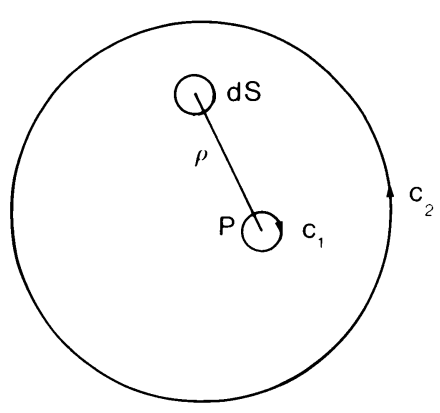

FIG. 2. Showing the point $P$ in the plane occupied by the moving plate and fixed relative to the arc.

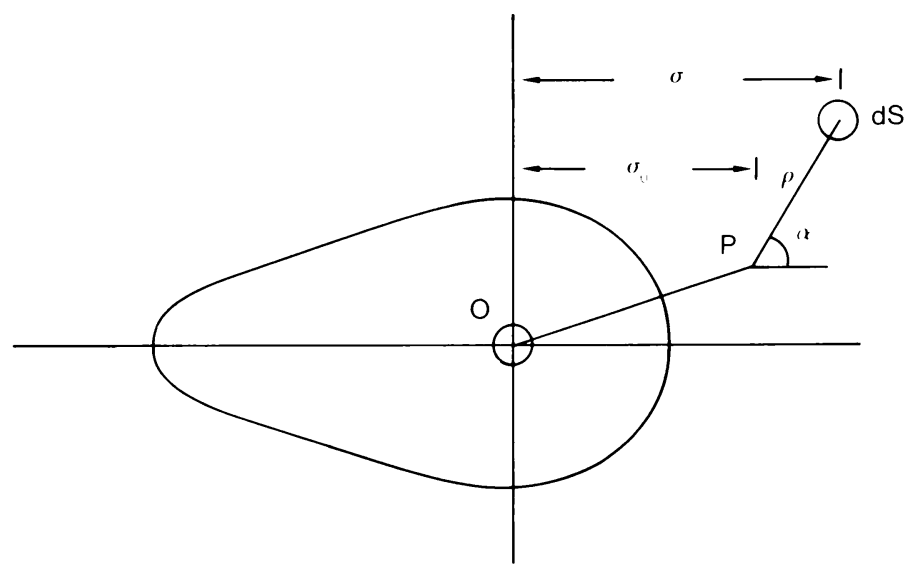

FIG. 3. Showing meaning of symbols $\sigma$ and $\sigma_{0}$ used in text.

whence, by Eq. (4), on letting $\sigma_{0}$ stand for the value of $\sigma$ at $P$, (Fig. 3)

$$
\Phi=\frac{1}{2 \pi \kappa h} \int_{S} W K_{0}(\lambda \rho v) e^{\lambda{ }^{\lambda(}\left(\sigma-\sigma_{0}\right)} d S .
$$

If we now move the point $P$ to 0 , the arc spot centre (Fig. 1), and denote by $\Phi_{0}$ the temperature at 0 then, since (Fig. 3)

$$
\sigma-\sigma_{0}=\rho \cos \alpha,
$$

where $\alpha$ is the angle that the radius from $P$ makes with - n (Fig. 3), we obtain

$$
\Phi_{0}=\frac{1}{2 \pi \kappa h} \int_{0}^{2 \pi} \int_{0}^{\infty} W K_{0}(\lambda \rho v) e^{i \rho v \cos \alpha} \rho d \rho d \alpha .
$$

Using Bessel's integral we readily obtain

$$
2 \pi I_{0}(t)=\int_{0}^{2 \pi} e^{t \cos \pi} d \alpha
$$

and so

$$
\Phi_{0}=\frac{1}{\kappa h} \int_{0}^{\infty} \rho W I_{0}(\lambda \rho v) K_{0}(\lambda \rho v) d \rho .
$$


4. Cathode arc spot temperature. The formula for $W$ is [2]

$$
W=\frac{E I}{4 \pi}\left(\frac{1}{\sqrt{\left(\rho^{2}+s_{1}^{2}\right)}}-\frac{1}{\sqrt{\left(\rho^{2}+s_{2}^{2}\right)}}\right),
$$

where $I$ is the current in the external metallic circuit of the arc, $E$ is the axial electric field along the arc column, and $s_{1}$ and $s_{2}$ are the distances from the arc spot centre, 0 , to the lower and upper terminations of the radiating portion of the arc column. By substituting from this equation into Eq. (9), and defining a function $F$ by

$$
F(\mu)=\int_{0}^{\infty}\left(\frac{1}{t}-\frac{1}{\sqrt{\left(t^{2}+\mu^{2}\right)}}\right) t I_{0}(t) K_{0}(t) d t
$$

we obtain, using Eqs. (3) and (9)

$$
\Phi_{0}=\frac{E I}{2 \pi \rho c v h}\left(F\left(\lambda v s_{2}\right)-F\left(\lambda v s_{1}\right)\right) \text {. }
$$

5. Results. The function $F(\mu)$ just defined was evaluated by numerical integration; the contributions to the integral for very small and very large values of the argument of the integrand being found analytically. Tables of Bessel functions and Simpson's rule were used to carry out the numerical work.

Watson's tables [7] cover a range of arguments starting with 0.02 and so $F(0.02)$ was evaluated using the series [8]

$$
\begin{gathered}
K_{0}(t)=-I_{0}(t)\{\ln (t / 2)+0.5772 \cdots\}+(t / 2)^{2}+\cdots, \\
I_{0}(t)=1+(t / 2)^{2}+\cdots .
\end{gathered}
$$

In fact the product $I_{0}(0.02) K_{0}(0.02)$ found from Watson's tables differs from the value of this product found, using the above expressions, with terms in $t^{2}$ and above omitted, by only $0.02 \%$. Consequently the value of $F(0.02)$ was determined analytically using the approximation

$$
I_{0}(t) K_{0}(t) \simeq-\{\ln (t / 2)+0.5772\} .
$$

On the other hand [7]

$$
\begin{aligned}
& \lim _{t \rightarrow \infty} K_{0}(t)=\frac{e^{t}}{(2 \pi t)^{1 / 2}}, \\
& \lim _{t \rightarrow \infty} I_{0}(t)=\frac{\pi^{1 / 2}}{2 t} e^{-t},
\end{aligned}
$$

and these expressions were used to evaluate analytically the contribution to the integral $F(\mu)$ for values of $t$ at which $I_{0}(t) K_{0}(t)$ approximated $1 / 2 t$; the asymptotic value of this product. 
Over the intermediate range of $t$ values, where integrations were carried out by use of Simpson's rule, coarse and fine intervals were chosen to ensure sufficient accuracy for purposes of graphical presentation of the results.

For iron $\lambda$ equals $6.8 \times 10^{4} \mathrm{~s} / \mathrm{m}^{2}\left(0^{0} C\right)$ [9] and, for an arc traversing an iron plate at $0.1 \mathrm{~m} / \mathrm{s}, \mu$ may have values as small as 0.1 or as large as 50 . Values of $\mu$ in this range were chosen to develop the function $F(\mu)$, shown graphically in Figs. $4 a$ and $4 b$.

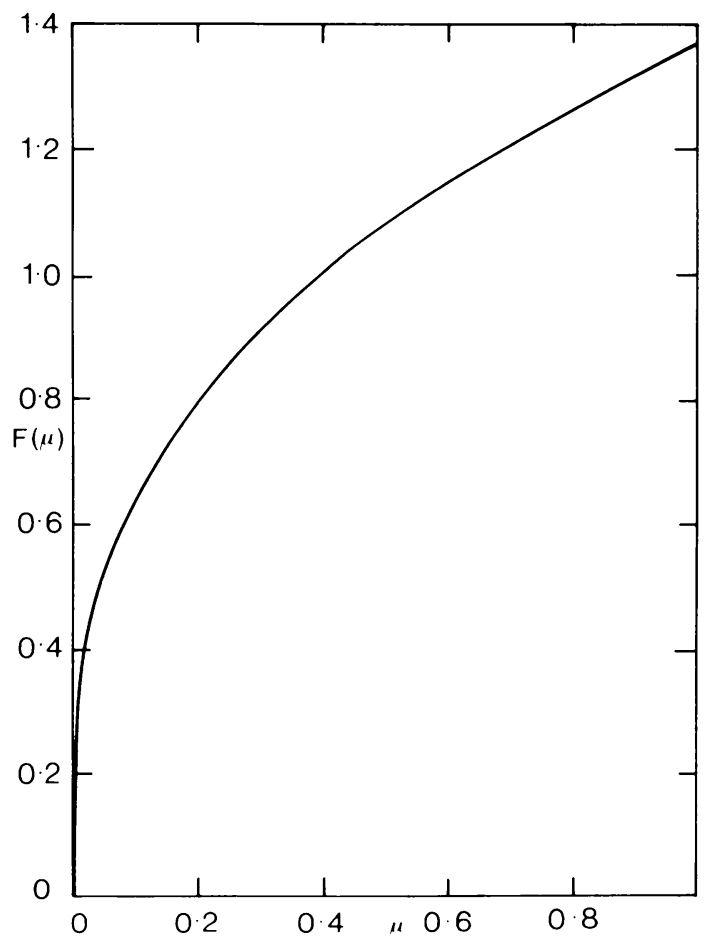

FIG. 4a. The function $F(\mu)$ over the range of values in which $\lambda v_{1}$ generally lies.

6. Discussion and conclusions. Equation (10) only remains applicable if the speed of traverse of the arc is high enough so that temperatures developed in the plate remain small; since otherwise the heat flow problem may become nonlinear, owing to the temperature dependence of the thermophysical constants [9]. Moreover Eq. (10) applies only if all of the radiation received by the plate is absorbed. Consequently in experimental applications it is necessary to use oxidised plates, or plates treated with lamp black.

Under these conditions values of $E, I, v$ and $s_{2}$ (approximately equal to the gap distance) may be easily measured and the arc spot temperature, $\Phi_{0}$, may also be readily measured, by one of several methods. These measurements used in conjunction with Eq. (10) and Fig. 4 then enable the value of $s_{1}$, the size of the cathode dark space, to be calculated, for a variety of metallic arcs. 


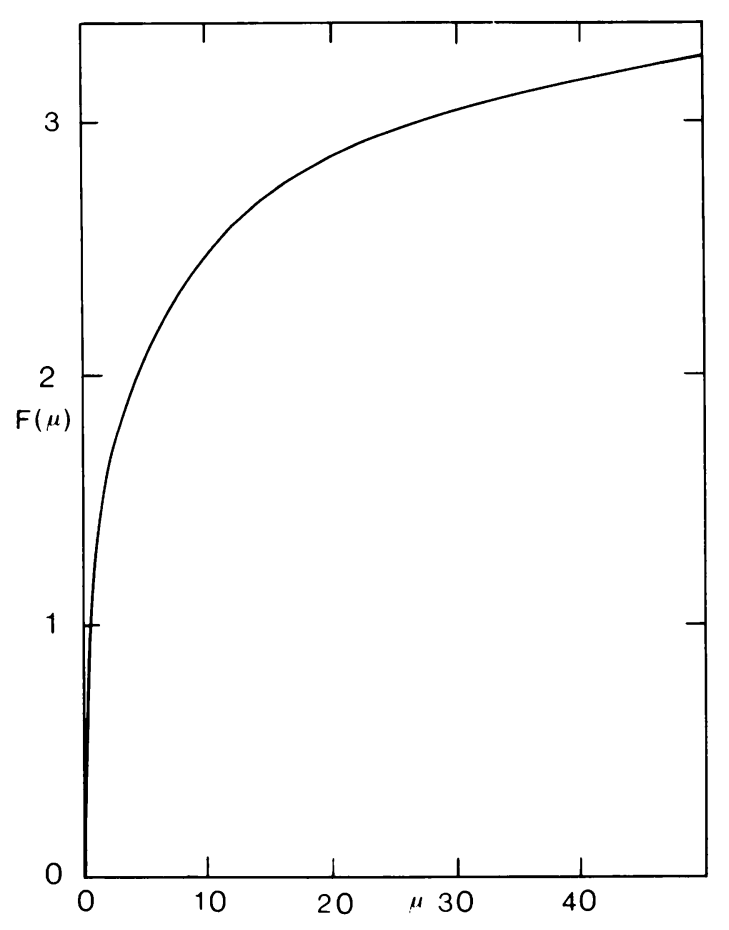

Fig. 4b. The function $F(\mu)$ over that range of $\mu$ values in which $\lambda u s_{2}$ generally lies.

\section{REFERENCES}

[1] J. M. Somerville, The Electric Arc, Methuen, London, (1959) p. 57

[2] R. L. Bish, Heat flux density distribution on the electrodes of an arc, Quart. Appl. Math. 47, 379-383 (1989)

[3] H. A. Wilson, On heat convection, Proc. Cambridge Phil. Soc. 12, 406-423 (1904)

[4] D. Rosenthal, Mathematical theory of heat distribution during cutting and welding, Welding J. 20, 220-234 (1941)

[5] B. Spain, Vector Analysis, Van Nostrand, 1965, pp. 66, 68

[6] M. G. Smith, Introduction to the Theory of Partial Differential Equations, Van Nostrand, 1967, p. 113

[7] G. N. Watson, A Treatise on the Theory of Bessel Functions, Cambridge University Press, 1945 pp. $202,698-713$

[8] H. S. Carslaw and J. C. Jaeger, Operational Methods In Applied Mathematics, Oxford University Press, 1941, p. 350

[9] G. W. C. Kaye and T. H. Laby, Tables of Physical and Chemical Constants, Longmans, 1936, pp. $54,56,84$ 